\title{
A Experiência da Enfermidade: Considerações Teóricas
}

\section{Experiencing Illness: Theoretical Considerations}

\author{
Paulo César Alves ${ }^{1}$
}

\begin{abstract}
ALVES, P. C. Experiencing Illness: Theoretical Considerations. Cad. Saúde Públ., Rio de Janeiro, 9 (3): 263-271, jul/sep, 1993.

This paper intends to establish some definitions and deal with key concepts and terms related to the understanding of illness as an experience. At the theoretical level, it aims to analyze the individual and collective determination of the experience of illness as the mediation between the macroscopic universe of meaning established in society and the ways in which this universe is sujectively real to individuals. This inquiry starts with the supposition that illness as an experience is both a subjetive and intersubjetive reality.
\end{abstract}

Key words: Illness Experience; Culture and Illness; Illness Behavior; Career of Illness

\section{INTRODUÇÃO}

As crenças e valores médicos constituem respostas socialmente organizadas para a doença. Refletem o complexo interativo entre grupos sociais, instituições, padrões de relacionamento e um corpo específico de conhecimento. Este complexo tem sido tradicionalmente estudado pelos sociólogos e antropólogos como formando uma realidade subjetivamente dotada de sentido pelos membros da sociedade. Cabe ao pesquisador, através de instrumentos teórico-metodológicos apropriados, esclarecer esta realidade. Estas construções teóricas, contudo, têm sido problemáticas.

Os estudos dos fatores socioculturais que configuram as crenças e valores médicos dos indivíduos não têm sido satisfatoriamente consistentes e sistemáticos. Há pouca concordância e muita polêmica sobre o significado dos termos e conceitos usados pelos estudiosos da Sociologia e da Antropologia Médica. A idéia de "experiência da enfermidade" é, neste aspecto, um exemplo significativo. Como uma categoria analítica, "experiência da enfermidade" é um termo que se refere aos meios pelos quais

${ }^{1}$ Centro de Estudos Etno-Epidemiológicos e SócioAntropológicos da Saúde. Departamento de Sociologia, Universidade Federal da Bahia. Rua Padre Feijó, 29. Salvador, BA, 40110-170, Brasil. os indivíduos e grupos sociais respondem a um dado episódio de doença. Para analisar este conceito, a literatura especializada parte da premissa de que as pessoas (re)produzem conhecimentos médicos existentes no universo sociocultural em que se inserem. Todavia, a interpretação de como os indivíduos se apropriam de um conjunto de idéias e crenças relativas à doença tem sido problemática para a Antropologia.

Podemos agrupar as diversas teorias que procuram explicar este fenômeno em dois grandes modelos interpretativos ou perspectivas analíticas. Basicamente, estes modelos diferem entre si na ênfase dada à determinação social ou a estruturas cognitivas sobre a enfermidade. Por um lado, autores como Herzlich (1973), Taussig (1980), Frankenberg (1980), Comaroff (1982), Stacey (1986) e Unschuld (1986) exploram mais diretamente a premissa de que as forças e relações sociais configuram o conhecimento, as crenças e a escolha de tratamento da enfermidade. Por outro lado, embora sem negar explicitamente esses determinantes, Good (1977) e um grupo de antropólogos associados com a etnografia clínica (Kleinman et al., 1978) têm enfatizado as estruturas cognitivas subjacentes aos relatos individuais sobre a doença.

Estas perspectivas são significativas em si mesmas e não estabelecem, necessariamente, pressupostos antagônicos entre si. Acreditamos 
que, para analisar a experiência da enfermidade, não temos que escolher entre os dois modelos propostos. Esta experiência, em si mesma, desvela aspectos tanto sociais como cognitivos, tanto subjetivos (individuais) como objetivos (coletivos). A questão chave que deve ser colocada é como tornar "inteligível" o movimento pelo qual as práxis individuais e a generalidade do social se constituem e reconstituem entre si.

Tendo em vista esta questão, o presente artigo propõe estabelecer considerações que possam elucidar os processos pelos quais os indivíduos se apropriam e integram subjetivamente formas convencionais da vida sociocultural. Mais especificamente, procuramos examinar algumas premissas teóricas necessárias para o estudo da experiência da enfermidade enquanto uma realidade construída por processos significativos intersubjetivamente partilhados. Tomando em consideração este objetivo, estabeleceremos alguns conceitos chaves e termos relevantes das literaturas sociológica e antropológica relacionados à questão da saúde e da enfermidade. É desnecessário dizer que não pretendemos fazer um levantamento exaustivo desta bibliografia, nem tampouco oferecer um modelo explicativo conclusivo sobre como os indivíduos respondem a episódios concretos de doença.

Subjacente à nossa proposta de análise, acreditamos contribuir parcialmente para um dos mais instigantes movimentos no seio da Antropologia contemporânea: compreender as dimensões cognitivas e sociais incorporadas nas representações individuais. Os antropólogos sempre foram sensibilizados pela dimensão simbólica da cultura. A Antropologia, todavia, ainda não encontrou, de forma satisfatória, os fundamentos teóricos que lhe permitam responder como os processos cognitivos transformam as experiências subjetivas em realidades dotadas de significação. Responder a esta questão, a nosso ver, significa conciliar a visão de cultura, entendida como parâmetro ou quadro de referência que governa a atividade humana, com os processos e formas sociais subjacentes às representações mentais.

\section{COMPORTAMENTO DO ENFERMO:}

\section{A EMERGÊNCIA DE UM CONCEITO}

Um dos primeiros estudos sobre a experiência da enfermidade foi desenvolvido por Talcott Parsons (1951). A doença, para este autor, constitui um desvio e deve ser analisada como um resultado da inter-relação, determinada por direitos e deveres, entre o doente e o terapeuta. Assumindo a sua condição de doente, isto é, adotando o papel de enfermo (sick role), o indivíduo passa a não ser mais considerado responsável pelo seu estado e fica legitimamente isento das obrigações sociais normais, desde que procure ajuda competente e coopere com o tratamento indicado. A enfermidade, portanto, não é meramente um estado de sofrimento, mas também uma realidade social.

Em que pese às críticas sobre a teoria de Parsons, devemos reconhecer que ela abriu um espaço significativo para os estudos que procuram analisar os meios pelos quais os indivíduos e grupos diferem entre si em suas respostas para a doença. Trabalhos realizados nos Estados Unidos, principalmente na década de 60 , procuraram mostrar que grupos sociais, como judeus e italianos, assumiam o papel de enfermo de formas diferenciadas. Tais estudos constituem o que, na literatura especializada, denomina-se "comportamento do enfermo" (illness behaviour).

Para Mechanic (1968), um dos primeiros teóricos a iniciar os estudos sobre esta temática, o termo "comportamento do enfermo" diz respeito ao processo pelo qual (1) a dor e os sintomas são definidos, dotados de significação e socialmente rotulados; (2) a ajuda é requisitada; e (3) o regime de vida é transformado.

É importante observar que estes estudos não chegaram a desafiar o conceito parsoniano do papel do enfermo. O foco da atenção, contudo, mudou. Passou-se de uma análise macrossociológica, totalizante, para um nível interpretativo voltado para comportamentos específicos de grupos sociais. Estes trabalhos, portanto, estavam dirigidos para o entendimento de como os sintomas são percebidos e avaliados por dife- 
rentes grupos ou tipos de pessoas em diferentes situações sociais. Dois grandes modelos são encontrados nestes estudos: o individualista e o coletivista. $\mathrm{O}$ primeiro modelo tendeu a reduzir o comportamento humano perante a enfermidade a questões "psicológicas". Exemplos deste modelo seriam os trabalhos de Rosenstock (1966), Mechanic \& Volkart (1961) e Kosa \& Robertson (1969). As análises de Zborowski (1952) e Zola (1973), por outro lado, enfatizam as diferenças nos valores e atitudes relacionados à doença como resultantes das diferenças entre as classes sociais, faixas etárias e etnias. Nesta abordagem coletivista, o papel dos fatores culturais torna-se a parte central da investigação.

Os estudos tradicionais do comportamento do enfermo apresentam alguns problemas teóricometodológicos. Devemos observar que tratavam-se de trabalhos voltados a identificar e explicar fatores de baixa ou alta utilização dos serviços médicos profissionais (McKinlay, 1972). Objetivando entender os fatores sociais que pudessem influenciar os padrões de uso dos serviços de saúde, estes estudos centraram seu universo de análise em pessoas que estavam ou estiveram sob tratamento médico e, portanto, que já tinham tomado a decisão de seguir uma forma de tratamento. Quase nada disseram sobre como e por que as decisões foram tomadas. Conseqüentemente, pouca atenção foi dada aos diferentes cursos de ação adotados antes da consulta ou do tratamento. Além do mais, como observa Dingwall (1976), tais estudos prenderam-se ao modelo biomédico como o único capaz de explicar as experiências da aflição. Uma grande exceção foi o trabalho de Eliot Freidson (1988).

Uma das principais contribuições de Freidson (1988) para a discussão do comportamento do enfermo foi a formulação do conceito de "sistema leigo de referência". Este conceito, em alguns aspectos, supera alguns problemas colocados pela teoria de Parsons. Estabelecendo uma distinção entre processos biológicos e as respostas sociais para este processo, Freidson desenvolveu a idéia de um sistema pluralístico de valores para dar conta das diversas definições de "doença". O modelo biomédico concebe a doença como uma propriedade física do comportamento, isto é, como resultado de fatores eminentemente morfo-fisiológicos. Trata-se de um modelo dominante na nossa sociedade, devido ao processo social pelo qual os médicos obtiveram o monopólio em estabelecer a jurisdição exclusiva sobre a definição de doença e tratamento. Este modelo, contudo, não leva em conta os significados sociais atribuídos ao comportamento do enfermo. Assim, "enquanto o médico pode usar a ciência biofísica para explicar os sinais que ele rotula como doença, ele enquanto tal não pode explicar o comportamento do doente pela referência a esta ciência" (Freidson, 1988). A doença deve ser vista como um desvio social e ser analisada pelo sistema leigo de referência, isto é, pelo corpo de conhecimentos, crenças e ações, através do qual ela é definida pelos diversos grupos sociais. Tal sistema está baseado em premissas que diferem do modelo biomédico e é resultante de estruturas sociais específicas de cada sociedade.

Pela compreensão deste sistema, observa Freidson (1988), é que podemos entender o processo de escolha de tratamento, pois, ao ser socialmente definido como enfermo, o indivíduo desencadeia uma seqüência de práticas destinadas a uma solução terapêutica. Freidson chama este processo career of illness, que traduziremos livremente como "itinerário terapêutico". Por este itinerário os indivíduos podem ter experiências com as várias agências de tratamento, ficando, assim, legitimados a assumirem um papel de enfermos, pois cada uma delas tem um caráter de imputabilidade ao atribuir suas próprias noções terapêuticas.

Embora seja uma abordagem mais elaborada para a compreensão social da doença, a teoria de Freidson (1988) negligencia questões importantes. Trata-se de uma teoria que, por enfatizar exclusivamente a existência de estruturas cognitivas padronizadas em determinados grupos sociais, nada nos diz sobre o modo pelo qual os indivíduos empregam o seu saber médico. $\mathrm{O}$ conceito de sistema leigo de referência não explica como surgem as expectativas sociais envolvidas com a enfermidade e por que elas diferem entre indivíduos pertencentes a um mesmo grupo social. Este conceito é apresentado por Freidson de forma auto-evidente, como um fenômeno externo aos indivíduos. De acordo com esta perspectiva, o doente é um ser que 
responde "passivamente" à configuração estrutural de uma dada rede de referência leiga. Os atores sociais de Freidson, como argúi Dingwall (1976), "não criam e usam sua cultura, mas são criadas e usadas por ela".

É inegável que tanto Parsons (1951) como Freidson (1988) contribuíram significativamente para a compreensão da determinação social da experiência da enfermidade. Porém, ambos confinaram suas teorias a um alto nível de abstração. Por desenvolverem uma perspectiva com pretensões à universalidade objetiva, não puderam oferecer instrumentos mais operacionalizáveis para a análise de experiências e práticas concretas dos indivíduos. Ambos partiram do pressuposto de que o comportamento do doente expressa uma realidade sociocultural objetiva que deve ser decodificada. A cultura, para eles, resolve-se, em última instância, em uma estrutura - um código - que permanece oculta perante as ações concretas dos indivíduos. Assim, cabe ao pesquisador procurar a regularidade através da diversidade das ações humanas. Ou, em outras palavras, o que se pede ao cientista social é que desenvolva modelos interpretativos que possam dar conta das realidades determinantes subjacentes às versões subjetivas dos informantes locais. Trata-se, portanto, de uma perspectiva teórica que tende a admitir, implicitamente, procedimentos metodológicos conclusivos. Se a estrutura cultural é apreendida como algo pré-dado, ela é tanto previsível como dedutível, pois pela generalização concluímos o particular. Esta perspectiva de análise social chamaremos de estrutural.

A abstração e a decodificação, tomadas em seus extremos pela perspectiva estrutural, tendem a reduzir o mundo cultural a processos sem sujeitos, isto é, sem atores historicamente datados que interagem em contextos sociais específicos. A concepção superorgânica de cultura subjacente à perspectiva estrutural trata o conhecimento cultural como uma forma de "consenso" em que os mundos de significados são reduzidos a agregados estatísticos de opiniões ou valores individuais. Neste aspecto, tomando-se esta perspectiva isoladamente, o pesquisador perde o contato com as diversidades e idiossincrasias humanas sobre os quais repousam as "realidades estruturantes" do mundo social. Não se leva em conta que as estruturas cognitivas são instrumentos do entendimento, atualizados através de enfoques performativos concretos de indivíduos e grupos sociais, e não meramente saberes proposicionais tipificados e estereotipados. Devemos observar que tais estruturas formam complexos processos de interpretação pelos quais os indivíduos, em suas contingências históricas e biográficas, atribuem significados às suas aflições.

\section{O CONCEITO DE MODELO EXPLICATIVO}

Trabalhos sócio-antropológicos mais recentes têm procurado superar os problemas teóricometodológicos dos estudos tradicionais do comportamento do enfermo. Dentre esses trabalhos, destacaremos apenas os que se relacionam com o conceito de "modelo explicativo" (ME).

Esse conceito foi inicialmente desenvolvido por Byron Good (1977) em "The heart of what's the matter: Semantics and illness in Iran". O trabalho de Good foi, em grande parte, responsável pela formação de um grupo de pesquisadores preocupados com a etnografia clínica. Kleinman (1981), um dos mais influentes representantes deste grupo, define modelo explicativo da enfermidade como "as noções sobre um episódio de doença e seu tratamento que são empregadas por todos aqueles engajados em um processo clínico". Assim, o modelo explicativo é um conjunto de proposições ou generalizações, explícitas ou tácitas, sobre a enfermidade.

Ao explicarem a enfermidade, os modelos explicativos "determinam o que é considerado como evidência clínica relevante e como esta evidência é organizada e interpretada para abordagens racionalizadas de tratamento específico" (Kleinman, 1981). Estruturalmente, estes modelos diferem entre si na resposta que dão à etiologia, ao tempo e modo do aparecimento de sintomas, à patofisiologia, ao curso da doença (grau de severidade) e ao tratamento.

Trata-se de um conceito que objetiva estabelecer um quadro analítico capaz de conduzir comparações entre culturas e sistemas de tratamento. Os MEs da enfermidade são essencialmente vistos como estando ligados a conjuntos específicos de conhecimentos e valores funda- 
mentados nos diferentes setores do sistema médico. A comparação entre estes setores (assim como a análise de suas inter-relações) é essencialmente um estudo dos seus diferentes modelos explicativos. Como diz Kleinman (1978), "os relacionamentos no cuidado à saúde (por exemplo, as relações pacientefamília ou paciente-terapeuta) podem ser estudados e comparados como transações entre diferentes MEs e os sistemas cognitivos $e$ posições na estrutura social nos quais eles estão ligados".

Como regra, os teóricos dos MEs observam que os subsetores de um sistema médico são compartimentalizados: "certos tipos de prática são mais adequadas a explicar e, portanto, curar certos tipos de doença" (Harrel, 1991). Desta forma, em um contexto pluralístico, os subsistemas médicos tendem a se tornar ligados a doenças específicas, de tal forma que os padrões de procura de auxílio (pathways of resort) podem ser delineados por diferentes tipos de situações de enfermidade.

A idéia dos modelos explicativos é um poderoso instrumento teórico que permite explorar questões como aderência a tratamentos, escolha e avaliação de terapias. Acreditamos, contudo, que o conceito de ME responde apenas parcialmente à questão da experiência da enfermidade. Os teóricos dos MEs tendem a explicar os conhecimentos e crenças médicas em termos de um conjunto singular de estruturas cognitivas subjacentes aos setores do sistema médico. $\mathrm{Na}$ procura de uma lógica interna dos significados atribuídos à enfermidade, e dentro de um interesse essencialmente clínico, Kleinman e seus seguidores não dão a devida atenção ao fato de, geralmente, as pessoas atribuírem, ao mesmo tempo, diferentes interpretações para as suas aflições. As percepções, crenças e ações dos indivíduos são geralmente heterogêneas, complexas e ambíguas.

É importante observar que as crenças, valores e práticas existentes em um sistema médico não estão constituídas necessariamente por categorais lógicas coerentes. As incoerências e contradições tornam-se, muitas vezes, mais visíveis no contexto de um sistema médico pluralístico, com realidades clínicas muitas vezes conflitantes. Nestes contextos, os diversos subsetores terapêuticos usualmente não estabelecem fron- teiras definidas entre si. Embora não formem uma totalidade funcionalmente integrada, os subsetores podem ser interconectados de diversas formas. Assim, eles podem coexistir com pouca capacidade de excluirem-se mutuamente. Por outro lado, é também importante observar, como chama a atenção Comaroff (1978), que as relações entre as interpretações subjetivas dos indivíduos e os modelos explicativos dos sistemas médicos não são necessariamente enquadradas de acordo com um modelo integrativo e coerente. Conforme observa Last (1981), em um sistema médico pluralista, as pessoas podem se engajar em processos terapêuticos sem saber ou querer saber sobre a lógica interna do sistema escolhido. Para este autor, sob certas condições, o não saber ou não querer saber pode-se tornar institucionalizado como parte de uma cultura médica.

A interpretação que as pessoas elaboram para uma dada experiência de enfermidade é o resultado dos diferentes meios pelos quais elas adquirem seus conhecimentos médicos. Tais conhecimentos são diferentes entre as pessoas, por serem originados em situações biográficas determinadas. Conseqüentemente, os conhecimentos médicos construídos pelos membros ordinários da sociedade devem ser "localizados" em um contexto compreensivo mais amplo do que aquele oferecido pelos diversos subsetores de um sistema médico.

O conhecimento médico de um indivíduo tem sempre uma história particular, pois é constituído de e por experiências diversas. Assim, é de se esperar que este conhecimento exista em um fluxo contínuo e quo mesmo seja passível de mudanças, tanto em termos de extensão como em termos de estrutura. A interpretação da enfermidade tem uma dimensão temporal não apenas porque a doença, em si mesma, muda no decorrer do tempo, mas também porque a sua compreensão é continuamente confrontada por diferentes diagnósticos construídos por familiares, amigos, vizinhos e terapeutas. $\mathrm{O}$ conhecimento médico de um indivíduo está continuamente sendo reformulado e reestruturado, em decorrência de processos interativos específicos. Assim, como argumenta Young (1981, 1982), é esperado que o indivíduo produza mais do que um tipo de explicação sobre sua enfermidade, porque seu conhecimento é 
sempre recorrente e processual. Conseqüentemente, os modelos explicativos da enfermidade são apenas uma entre as diversas formas de conhecimento médico, não estando os mesmos necessariamente implícitos em todas as expressões que os indivíduos fazem sobre as suas aflições. Como nenhum conjunto singular de estruturas cognitivas pode ser tomado como fonte última das expressões do indivíduo, nenhuma forma é, a priori, o autêntico conhecimento dos fatos médicos do indivíduo.

\section{CONSIDERAÇÕES FINAIS}

Nas páginas precedentes, procuramos examinar alguns pontos básicos da literatura sócioantropológica que são pertinentes ao entendimento da questão da enfermidade. Ao apresentá-los de forma um tanto sistemática, podemos observar que estes estudos não estabelecem de forma satisfatória os fundamentos teóricos que lhes permitam responder como os processos cognitivos sobre a enfermidade são socialmente construídos. Para respondermos a esta questão, a nosso ver, é necessário descermos ao nível dos microfundamentos da experiência da enfermidade, para que possamos atingir o domínio dos macroprocessos sociais (padrões de comportamento, quadros culturais de referência, instituições, etc.). Assim, sem se perder em um "torvelinho de relativismo cultural", torna-se necessário "descer aos detalhes, além das etiquetas enganadoras, além dos tipos metafísicos, além das similaridades vazias, para apreender corretamente o caráter essencial não apenas das várias culturas, mas também dos vários tipos de indivíduos dentro de cada cultura " (Geertz, 1978).

Nas páginas seguintes, procuraremos estabelecer algumas premissas teóricas que julgamos serem necessárias para a compreensão da enfermidade. Evidentemente, esta análise não será exaustiva, mas esperamos que ela possa estimular a discussão crítica de se trabalhar com o conceito de experiência da enfermidade.

Acreditamos que o ponto de partida para a compreensão da enfermidade é que ela está necessariamente presa a uma experiência. É a experiência de sentir-se mal que, por um lado, origina, por si mesma, as representações da doença e, por outro lado, põe em movimento a nossa capacidade de transformar esta experiência em um conhecimento. É através das impressões sensíveis produzidas pelo mal-estar físico e/ou psíquico que os indivíduos se consideram doentes. Não poderíamos saber a priori que estamos doentes sem que a sensação de que "algo não vai bem" tenha sido revelada anteriormente. Assim, esta sensação constitui o primeiro passo para designar, de modo conveniente, o sentido da enfermidade (Telles \& Pollack, 1981). Neste aspecto, a enfermidade é um processo subjetivo.

Antes de prosseguirmos, é importante ressaltar que nem toda alteração ou disfunção orgânica ou psíquica desperta necessariamente uma experiência de sentir-se mal. Processos ou estados patológicos podem estar presentes no nosso corpo sem que tenhamos consciência deles. As perturbações fisiológicas, tomadas em si mesmas, constituem o objeto da investigação biomédica. Só quando transformada em sintomas, em impressões sensíveis, é que a doença torna-se uma enfermidade.

A enfermidade, por outro lado, é mais do que uma situação emotiva decorrente de uma reação corporal. Ela estende-se para além dos limites do mundo sensível. Se a enfermidade se inicia com a experiência, isso não prova que toda ela derive da experiência. $O$ corpo humano, durante um processo patológico, pode fornecer um somatório de informações heterogêneas, como dor de cabeça, vômito, febre, fraqueza, etc. Cada informação constitui um "fato" isolado e, portanto, fechado. Uma dor de cabeça, por exemplo, é uma simples informação entre tantas outras e, em si mesma, nunca permitirá compreender outra coisa que não ela própria. Tomadas isoladamente, as simples informações não significam quase nada. Por outro lado, não é pela percepção de uma miríade de sensações coligadas que podemos garantir a validade da nossa apreensão da enfermidade. A multidão de experiências diversas precisa ser organizada em uma totalidade sintética, isto é, em configurações globais dotadas de sentido. Neste aspecto, a idéia de enfermidade não aponta apenas para as impressões sensíveis, e sim para o sentido atribuído a elas. Torna-se necessário, portanto, circunscrever as reações corporais em sistemas significantes. 
Para que a "matéria bruta" das impressões sensíveis seja organizada é necessário que ela seja "apreendida", isto é, fundamentada pela "tomada de consciência" de uma condição. Tomar consciência de uma dada situação é organizar um somatório de fatos em algo compreensivo e, portanto, significativo. A sensação de sentir-se mal encontra-se intrinsecamente acompanhada de uma compreensão do seu significado. Significar, como observa Sartre (1972), "é indicar outra coisa, indicá-la de tal forma que desenvolvendo a significação se vá encontrar precisamente o significado". A enfermidade, neste sentido, constitui-se em uma interpretação e em um julgamento sobre a matéria bruta das impressões sensíveis produzidas pelo corpo. Enfermidade não é um fato, mas significação.

A noção de significado, como enfatiza a filosofia hermenêutica, é sempre "significado para" alguém. O componente subjetivo da enfermidade, como visto, está fundamentado no ato individual de perceber uma experiência interior como problemática. Contudo, a construção do significado desta experiência não ocorre como um processo isolado. A consciência não constitui seus objetos ex nihilo, pela autonomia da interioridade subjetiva, mas a partir de processos interpretativos adquiridos na vida cotidiana. A enfermidade é subjetivamente dotada de sentido, na medida em que é afirmada como real para os membros ordinários da sociedade. É real porque é justamente originada no mundo do senso comum. Como observa Schutz (1973), o mundo da vida cotidiana funciona como um código de referência para os indivíduos. É importante lembrar que todo significado só é lógico para o indivíduo porque é socioculturalmente legitimado pelos seus semelhantes.

A produção dos significados é resultante não de um instante pontual do "eu", mas de toda uma história do "eu". Uma história que, necessariamente, constitui-se por processos de interação e comunicação com os outros. No dizer de Husserl, a consciência é, para um indivíduo, a possibilidade que tem o sujeito de constituirse em objeto para si mesmo e para os demais. $\mathrm{O}$ uso de vocabulários e técnicas apropriadas de representação legitima socialmente o significado que o indivíduo atribui à sua experiência afliti- va. Telles \& Pollack (1981), por exemplo, observam que o processo através do qual os indivíduos interagem com outros, no curso da enfermidade, envolve quatro estágios: “a) os outros sugerem, verificam ou negam que a pessoa está doente; b) ou outros indicam que tipo ou quantidade de sentimentos são aceitáveis; c) os indivíduos encontram meios para demonstrar a validade dos seus sentimentos, um processo para o qual outros contribuem; $e d$ ) outros dirigem o indivíduo para os legitimadores oficiais". Neste sentido, que podemos dizer que a consciência de sentir-se mal é sempre uma "consciência em situação", pois está relacionada com projetos e contextos existenciais específicos. Assim, para uma compreensão adequada da enfermidade, deve-se levar em conta tanto seus aspectos subjetivos, o que determina um mundo de diferenças interpretativas, como sues aspectos intersubjetivos, o que a torna "objetiva" para os outros.

Ao afirmarmos o caráter intersubjetivo da enfermidade, pressupomos a existência de certos parâmetros ou quadros de referência graças aos quais é construído o significado da experiência da enfermidade. Estes quadros de referência são internalizados pelos indivíduos através de processos concretos de interação social. Neste aspecto, os padrões culturais que as pessoas utilizam para interpretar um dado episódio de doença são criações sociais, ou seja, são formados a partir de processos de definição e interpretação construídos intersubjetivamente. Só dentro das coordenadas estabelecidas pelo mundo intersubjetivo do senso comum é que a experiência da enfermidade é admitida como facticidade evidente por si mesma e compulsória.

\section{AGRADECIMENTOS}

Este trabalho não teria sido possível sem a importante colaboração da Dra. Miriam Rabelo e companheiros do CESAME, aos quais agradeço. 


\section{RESUMO}

ALVES, P. C. A Experiência da

Enfermidade: Considerações Teóricas. Cad. Saúde Públ., Rio de Janeiro, 9 (3): 263-271, jul/set, 1993.

O presente trabalho estabelece algumas definições e o tratamento de conceitos chaves e termos relevantes para a compreensão da experiência da enfermidade. Objetiva analisar, em um nível teórico, a determinação individual e coletiva desta experiência. A análise aponta para a relação entre o universo macroscópico de significados estabelecidos pela sociedade e o mundo subjetivo dos indivíduos. O trabalho parte do pressuposto de que a experiência da enfermidade é uma realidade subjetiva tanto como intersubjetiva.

Palavras-Chave: Experiência da Enfermidade; Cultura e Enfermidade; Comportamento do Enfermo; Itinerário Terapêutico

\section{REFERÊNCIAS BIBLIOGRÁFICAS}

COMAROFF, J., 1978. Medicine and culture: Some anthropological perspectives. Social Science and M edicine, 12B: 247-254.

1982. Medicine: symbol and ideology. In: The Problem of Medical Knowledge (Wright \& Treacher, eds.), pp. 75-91, Edinburgh: Edinburgh University Press.

DINGWALL, R., 1976. A spects of IIIness. London: Martin Robertson.

FRANKENBERG, R., 1980. Medical anthropology and development: A theoretical perspective. Social Science and Medicine, 14B: 197-207.

FREIDSON, E., 1988. Profession of Medicine. $2^{\text {nd }}$ ed., Chicago: The University of Chicago Press.

GEERTZ, C., 1978. A Interpretação das Culturas. Rio de Janeiro: Zahar.

GOOD, B., 1977. The heart of what's the matter: Semantics and illness in Iran. Culture, Medicine and Psychiatry, 1: 25-28.

HARREL, S., 1991. Pluralism, performance and meaning in Taiwanese healing; A case study. Culture, Medicine and Psychiatry, 15: 45-68.

HERZLICH, C., 1973. Health and IIIness: A Social Psychological Analysis. London: Academic Press.
KLEINMAN, A., 1978. Concepts and model for the comparison of medical systems as cultural systems. Social Science and M edicine, 12B: 85-94. 1981. Patients and Healers in the Context of Culture. Berkeley: University of California Press.

KLEINMAN, A.; EISENBERG, L. \& GOOD, B., 1978. Culture, illness and care: Clinical lessons from anthropological and cross-cultural research. A nnals of Internal M edicine, 88: 251-258.

KOSA, J. \& ROBERTSON, L. S., 1969. The social aspects of health and illness. In: Poverty and Health (J. Kosa \& T. Zola, eds.), pp. 40-79, Cambridge: Harvard University Press.

LAST, M., 1981. The importantance of knowing about not knowing. Social Science and M edicine, 15B: 387-392.

McKINLAY, J. B., 1972. Some approaches and problems in the study of the use of services: An overview. Journal of Health and Social Behaviour, 13: 115-152.

MECHANIC, D., 1968. Medical Sociology. New York: The Free Press.

MECHANIC, D. \& VOLKART, H., 1961. Stress, illness behavior, and the sick role. American Sociological Review, 26: 51-58.

PARSONS, T., 1951. The Social System. London: Routledge \& Kegan Paul Ltd.

ROSENSTOCK, I. M., 1966. Why people use Health Services. M ilbank Memorial Fund Quarterly, 64: 94-127.

SARTRE, J. P., 1972. Esboço de uma Teoria das Emoções. Lisboa: Editorial Presença.

SCHUTZ, A., 1973. Collected Papers, I. The Hague: Martinus Nijhoff.

STACEY, M., 1986. Concepts of health and illness and the division of labour in health care. In: Concepts of Health, IIIness and Disease (C. Currer \& M. Stacey, eds.), pp. 9-26, Berg: Leamington Spa.

TAUSSIG, M., 1980. Reification and the consciousness of the patient. Social Science and Medicine, 14B: 03-13.

TELLES, J. L. \& POLLACK, M. H., 1981. Feeling sick: The experience and legitimation of illness. Social Science and M edicine, 15A: 243-251.

UNSCHULD, P., 1986. The conceptual determination (Uberformung) of individual and collective experiences of illness. In: Concepts of Health, Illness and Disease (C. Currer \& M. Stacey, eds.), pp. 51-70, Berg: Leamington Spa.

YOUNG, A., 1981. The creation of medical knowledge: Some problems in interpretation. Social Science and M edicine, 15B: 379-386. 
1982. The Anthropologies of illness and sickness. Annual Review of Anthropology, 11: 257-285.

ZBOROWSKI, M., 1952. Cultural components in responses to pain. Journal of Social Issues, 8: 16-30.
ZOLA, I. K., 1973. Culture and symptoms: An analysis of patient's presenting complaints. In: Problems of Modern Society (P. Worsley, ed.), pp. 615-630, London: Penguin. 